\title{
La vertebración territorial en regiones de alta especialización: Valle Central de Chile \\ Alcances para el desarrollo de zonas rezagadas en torno a los recursos naturales
}

Alberto García-Huidobro. Arquitecto Pontificia Universidad Católica de Chile, Postgrado en Estudios Urbanos CIDU. Profesor Escuela de Arquitectura Universidad de Talca.

ANDRÉS MARAGAÑ O. Arquitecto Universidad de Los Lagos. Master en Urbanismo, UPC, Barcelona. Profesor Escuela de Arquitectura Universidad de Talca.

RESUMEN | Los procesos de reestructuración económica y la apertura de los mercados han generado múltiples y variadas consecuencias sobre nuestros territorios. Quizás para Chile, como para otros países latinoamericanos, uno de los más importantes procesos a nivel regional es el desarrollo de espacios altamente especializados o regiones-commodities. Distintos trabajos académicos señalan que interiormente, en conjunto con áreas de gran competitividad, se están generando áreas rezagadas o alejadas de los dinamismos económicos.

Por lo tanto: ¿En qué ha derivado su modelo de vertebración territorial y su sistema de centros poblados? ¿Cuáles son las características de estas áreas rezagadas? Y por ende, ¿cuáles son los desafíos a los cuales se enfrentan?

El Valle Central de Chile es una de estas áreas donde se evidencia dicha especialización. Por tanto, analizaremos la estructura física territorial en las que han derivado estas regiones y el papel que cumplen sus centros poblados en los nuevos escenarios. A partir de la caracterización de su vertebración territorial avanzaremos hacia la definición de sus zonas menos favorecidas, e intentaremos generar algunas hipótesis que respondan a sus desafíos y requerimientos futuros.

PALABRAS CLAVE | Desarrollo regional y local, desarrollo territorial, economía regional, integración territorial.

ABSTRACT | Economic restructuring processes and market opening have generated multiple and varied consequences in our territories. Perhaps for Chile, as with other Latin American countries, one of the most important processes at the regional level is the development of highly specialized spaces or commodity-regions. Different academic work indicates that within these spaces, as well as areas of high competitiveness, there are also backward areas with limited economic dynamism. Consequently: What has happened to the model of a territorial spine and a system of population centers? What are the characteristics of these backward areas? And what are the challenges to be faced? Chile's Central Valley is one of the areas that reveals this specialization. We analyze the physical structure of the territory that has been generated in these regions and the role that population centers play in these new scenarios. Based on a characterization of the territorial spine, we move towards a definition of most favoured areas and generate hypotheses relating to challenges and future requirements.

KEY WORDS | Regional and local development, territorial development, regional economy, territorial integration.

El presente escrito es parte de la investigación: "Los escenarios para una nueva organización del territorio, Valle Central de Chile" adscrito a la Dirección de Investigación (DI) de la Universidad de Talca, 2009.

Recibido el 30 de julio de 2009, aprobado el 4 de noviembre de 2009.

Correspondencia: Universidad de Talca - Escuela de Arquitectura, 2 Norte 685, Teléfonos (56) 71200200 - (56) 71201539 ,

Talca-Chile, Email: amaragaño@utalca.cl, ahuidobro@utalca.cl 


\section{La concentración ${ }^{1}$ y el sistema territorial chileno}

A partir de las últimas tres décadas ${ }^{2}$ la dinámica del modelo "territorial continental chileno" ha venido evolucionando en tres importantes procesos: la litoralización, la metropolización y la concentración de población en ciudades medias e intermedias ${ }^{3}$. El primero de estos procesos, concebido como el crecimiento de ciudades y asentamientos extendidos por las costas de Chile, se construye de forma disgregada y heterogénea ocupando la franja litoral del Pacífico ${ }^{4}$. La metropolización, por su parte, corresponde a la persistencia de la capital, Santiago ${ }^{5}$, por mantenerse como la gran metrópolis chilena. Lo anterior acontece a pesar de los cambios en el modelo económico que ha experimentado Chile en los últimos años, lo cual ha derivado en la concentración en la metrópolis de los principales nodos de las cadenas productivas, que en consecuencia ha provocado grandes desequilibrios en el territorio nacional (De Mattos, 2002).

El tercer proceso atañe a la concentración de población en algunas ciudades medias o intermedias del país, emplazadas tanto en el norte como en el sur de Chile. Este fenómeno, como los anteriores, también se verifica a partir de la estructuración económica, vale decir, la apertura del mercado y la implementación del modelo exportador. Lo anterior no resulta demostrable sólo en nuestro país, ya que también es posible constatarlo en otros países latinoamericanos, donde acontece un gran dinamismo en algunas ciudades de entre 100 mil a 300 mil habitantes. Incluso demuestran tasas de crecimiento superiores a las de la metrópolis del país respectivo (CEPAL, 1998; Pulido, 2003).

Este proceso en Chile corresponde a ciudades capitales regionales o provinciales, las cuales están situadas al interior de enclaves primoexportadores o regiones-commodities ${ }^{6}$ (Daher, 2003), que de alguna forma aspiran a convertirse en fuentes de equilibrio en el sistema total. Estas posiciones de fuerza parecen descansar en una especialización bastante profunda en la producción de las regiones chilenas, reflejada en una importante participación en el PIB nacional ${ }^{7}$ (Daher, 2003).

Es así como los procesos de concentración en estas ciudades están marcados por un gran dinamismo urbano, ligado a la expansión de la actividad terciaria de comercio

1 Usaremos la siguiente definición de concentración: "La concentración urbana es una determinada tendencia de la distribución espacial de la población, por la cual una ciudad de un sistema urbano determinado crece en población en forma sostenida en el tiempo a tasas superiores a las de crecimiento poblacional del conjunto de las ciudades del sistema". (Geisse, 1983, p. 49).

2 Nos referimos al cambio de modelo económico que sufre nuestro país, caracterizado por la apertura de los mercados y la implementación del modelo exportador a partir de las políticas llevadas a cabo desde 1973.

3 Según M. Gault (1989), en relación a la definición de ciudad intermedia, la noción de ciudad media hace alusión a aspectos estáticos: peso, población; en suma, a estadísticas, banalizando con ello, en alguna medida, su objeto. Insistir en la noción de ciudad "intermedia” es, por el contrario, señalar la importancia de aspectos dinámicos, la noción de estrategia, de lugar a conquistar, de espacio a construir.

4 Dentro de este proceso de concentración urbana en el espacio litoral se encuentran las ciudades y conurbaciones de segundo rango en Chile: Gran Valparaíso, La Serena-Coquimbo, Gran Concepción y Antofagasta, que son puertos ligados a la actividad exportadora. Por otro lado, aparece una gran cantidad de inversión inmobiliaria destinada a vivienda de segunda residencia, complejos turísticos y servicios.

5 La Región Metropolitana creció a un ritmo superior al resto del país, alcanzando una tasa de $8,1 \%$ anual entre 1990-1996, asimismo incrementó su contribución al PIB nacional, pasando de un 44,84\% en 1990 a generar el 47,45\% en 1997. Para 1992 la ciudad de Santiago tenía 4.295 .593 hab., mientras que para el último censo 2002 la Región Metropolitana de Santiago tiene 6.061.185, y el Gran Santiago 4.668.473, produciendo el 47,7 \% del PIB nacional (INE, 1992, 2002, 2004).

6 Según Daher (2003), "Las regiones-commodities son, por definición, regiones insertas plenamente en la economía global, ya que el mercado de los commodities es un mercado mundial. A diferencia de otras regiones productoras de bienes y servicios orientados al mercado local o a ámbitos regionales subglobales..." Finalmente sentencia: "Una Capital intensiva en capital y un resto de Chile intensivo en recursos naturales".

7 Aunque existen diferencias entre las regiones del norte y sur del país, "La participación en el PIB nacional de las regiones al sur de Santiago sumaba 31,56\% en 1975 y sólo $26,40 \%$ en 1997 . A su vez, en los mismos años las regiones al norte de la capital incrementaban su aporte del 23,46\% al 25,50\%". 
-transporte y servicios especializados-, la cual, junto con la construcción de viviendas, adquirieron un fuerte dinamismo durante estos años, incluso compensando el impulso de las actividades exportadoras (Sabatini \& Wormald, 2004). Según varios estudios realizados sobre ciudades intermedias en Chile, existe coincidencia en la importancia de éstas como centros administrativos regionales y provinciales, como centros prestadores de servicios para sus hinterlands y centros de transformación industrial de recursos naturales (Rovira, 2000; Romero \& Toledo, 2000; Mertins, 2000). De esta manera, podríamos asegurar que estas ciudades viven un despegue económico originado en su localización, donde se han convertido en unidades espacio-funcionales que atraen inversiones orientadas al mercado internacional -principalmente forestales, pesca, cultivos acuícolas y turismo especializado-, constituyéndose en verdaderos nodos en el sistema territorial chileno.

\section{Regiones-commodities y sus sistemas de centros poblados}

Quizás la principal característica de las regiones donde se emplazan estas ciudades, que de ahora en adelante llamaremos "ciudades regionales", es un crecimiento basado en la economía exportadora, sustentada y arraigada en su territorio, lo cual las ha hecho derivar en territorios altamente especializados: "Minerales, forestales o como la fruticultura fuera de temporada destinada a los mercados del hemisferio norte. Es decir, la mayoría de los productos considerados son comercializados antes de su transformación” (Szary, 1997, p. 66).

Así, estas regiones, que en los últimos años han mostrado una importante participación en el PIB nacional, muestran una inserción directa en la economía global, lo que configura un modelo distintivo y diferenciado con respecto a las dinámicas, por ejemplo, de la Región Metropolitana, cuya base económica es más diversificada. Lo que podría entenderse bajo la consigna: "el riesgo-región difiere radicalmente del riesgo-país" (Daher, 2003, p. 106). Bajo este perfil productivo, una de las principales características del sistema de centros poblados que vertebran el territorio de las regiones-commodities es la existencia de una estructura espacial polarizada ${ }^{10}$. Es decir, mientras las ciudades regionales demuestran sostenidos aumentos de población, altos grados de concentración económica y ambientes de aglomeración productiva, gran parte del territorio regional está compuesto por pequeñas agrupaciones: pueblos, caseríos, aldeas y villorrios, a partir de lo cual puede verificarse un patrón de distribución más bien disperso. A estos espacios rurales generalmente se les asocia menor cobertura o provisión de servicios, pues demandan mayor costo, menor acceso a la información -vital en tiempos de globalización- y mayor dificultad para atraer factores de producción; por tanto, menor acceso a la infraestructura productiva. Asimismo, las administraciones locales muestran sistemáticamente la imposibilidad de cubrir estas amplias zonas geográficas, lo que decanta en la antesala de la decadencia de su espacio (Arenas, 1999). Finalmente, el escenario que se figura para las regiones-commodities demuestra más bien regiones de subdesarrollo que territorios especializados y globales.

8 Nos parece más interesante resaltar este aspecto de estas ciudades.

9 Según este autor, la capital de Chile está profundamente orientada al MERCOSUR, mientras que el resto de Chile encuentra en Asia y Europa a sus principales socios comerciales.

10 En muchos trabajos académicos sobre regiones latinoamericanas, algunos de ellos nacidos de EE.UU., se muestra especial preocupación por un escenario fuertemente asimétrico y selectivo en cuanto al crecimiento territorial. Situación que enfrenta a las regiones centrales con las regiones periféricas. Asimismo, al interior de dichas regiones o áreas también aparecen asimetrías importantes, "regiones ganadoras vs regiones perdedoras", lo que aparece como producto del proceso globalizador (Fernández, 2007). 
Las causas de los procesos de concentración espacial, que están ligados a la distribución de los recursos en el territorio, muestran al mercado como el agente de mayor participación. Así, la aglomeración o concentración de actividades económicas refleja procesos de "causación acumulativa": "Las actividades se agrupan donde los mercados son grandes y los mercados se vuelven grandes donde las actividades se agrupan” (Krugman, 1996, p. 56). Incluso estos procesos, que se manifiestan en ambientes típicamente urbanos, hoy están dirigidos a todas las actividades económicas, ya no solamente a los sectores productivos (Camagni, 2005). Lo que significa, finalmente, la discriminación o selección de espacios según sus formas de integración económica, las cuales determinan algunos espacios dinámicos y otros en situación de subutilización o simplemente de abandono (Arenas \& Sabatini, 1994).

La aglomeración, por otro lado, genera una discriminación que, desde una mirada espacial al territorio, crea una distancia económica y social a partir de las distintas localizaciones (mayor distancia de la aglomeración, menor escala de producción). Así, en el caso de las regiones altamente especializadas, "la localización de los productos de mayor valor agregado, la infraestructura, la innovación y el poder adquisitivo se concentran focalizadamente en algunas ciudades, mientras los recursos naturales, de los cuales depende la región están dispersos por todo el territorio y generalmente es imposible trasladarlos, algunos son únicos o con ventajas comparativas claras" (CEPAL, 2006, p. 93).

En gran parte de Latinoamérica y en países en vías de desarrollo una gran cantidad de población habita en entornos menos favorecidos y lejos de alguna concentración, generalmente de manera dispersa en pequeñas localidades. Debido a las distancias económicas y las limitaciones de capital físico, humano y social, muchos de estos enclaves alejados son microempresas de supervivencia (Driven, 2002), lo que decanta en los llamados efectos "frontera" (CEPAL, 2006, p. 232).

Parte de las explicaciones de estos fenómenos se encuentra en el sendero evolutivo de cada territorio. Es así como en Chile estas regiones-commodities, históricamente, han ocupado gran parte del territorio, de forma de direccionar sus asentamientos hacia los recursos naturales; así, la dispersión de asentamientos facilita la explotación de dichos recursos, reconoce cierta diversidad y provee la necesaria mano de obra. Inicialmente configurado a través de la explotación colonial, luego por la exaltación de los enclaves latifundistas y, finalmente, mediante las políticas redistributivas de la propiedad de la tierra $^{11}$, se ha logrado sostener este modelo durante muchos años.

No obstante, los desequilibrios urbano-rurales parecen ser también una constante histórica, productiva y social, pues los niveles de concentración urbana, el crecimiento del capital industrial y la concentración espacial de las actividades de servicios especializados han favorecido a la urbanización, a pesar de los incrementos en la productividad rural. Y es que los grandes procesos migratorios parecen, incluso, producirse también en momentos de incrementos en las productividades territoriales de los recursos naturales (Geisser, 1983).

Finalmente, es así como Boisier reconoce que "sin desconocer los méritos de la lógica del mercado, también es conveniente reconocer que dicha lógica... no funciona según criterios territoriales sino de rentabilidad sectorial, cuyos efectos sobre el territorio tienen magnitudes y direcciones siempre discutibles" (Boisier, 1996, p. 69). La di-

11 Nos referimos a los procesos de reforma agraria, fundamentales en la comprensión de las políticas de modernización de las áreas rurales. 
námica que describe Boisier evidentemente manifiesta una pérdida de oportunidades y competitividad para las regiones, además de intensos desequilibrios internos y falta de equidad en el sistema total. Así se explican, de forma general, las inevitables asimetrías regionales que hoy están en curso, las cuales se hace urgente revisar.

\section{La vertebración ${ }^{12}$ territorial en regiones altamente especializadas. Una lectura de los intercambios y sus consecuencias}

\section{Valle Central de Chile}

A partir de lo anterior, es necesario avanzar en la comprensión de estos procesos sobre un área real, lo cual nos permitirá confirmar algunos de los rasgos antes propuestos y nos ayudará a construir algunas hipótesis sobre la estructura o modelo actual de los asentamientos en estos territorios. Por otro lado, nuestra intención es revisar las consecuencias más importantes que las nuevas dinámicas imprimen en el territorio y los posibles escenarios para una nueva organización de éste. El área de trabajo la llamaremos "Valle Central de Chile", de ahora en adelante VCC 13 (Figura 1).

FIGURA 1 | Valle Central de Chile y sus bordes

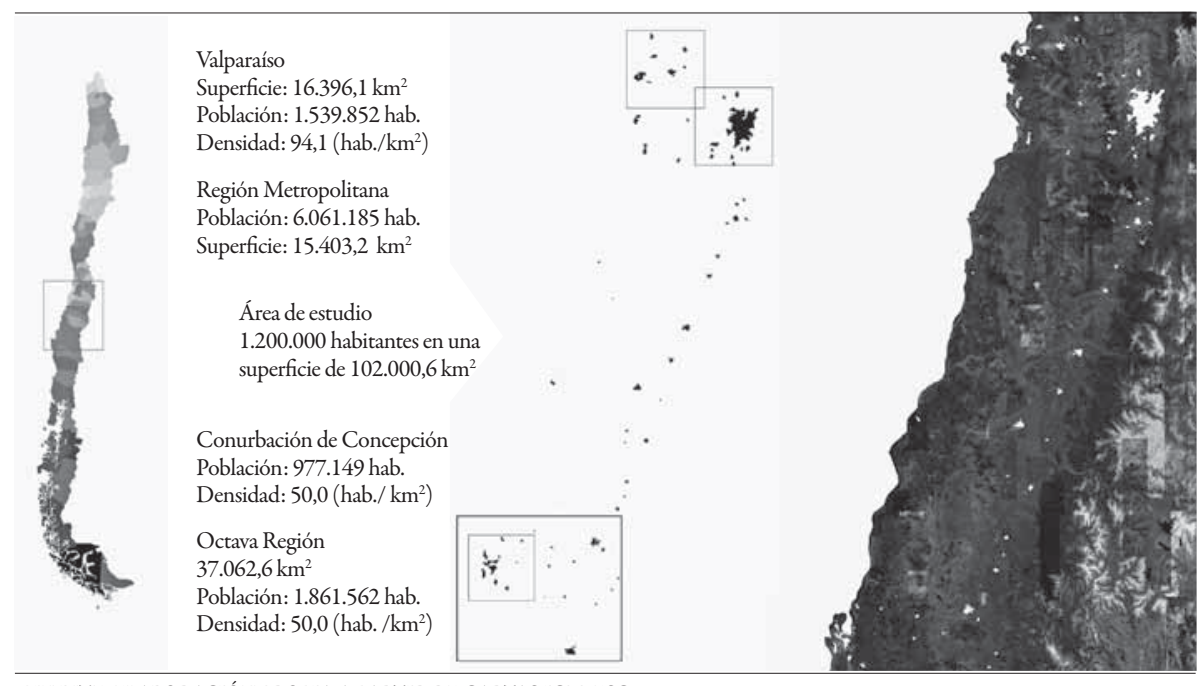

FUENTE ELABORACIÓN PROPIA A PARTIR DE CARTAS IGM, ESC: I:IO०.000

Este territorio es un valle de 400 kilómetros de largo, y está limitado por dos aglomeraciones que demuestran una base económica más diversificada, como lo son Santiago y Concepción. A partir de sus propias dinámicas y jerarquía en el sistema nacional, podrían bien servir de límites para una estructura territorial más bien homogénea: con un sistema de ciudades de tamaños y jerarquía similares, un territorio con rasgos geográfi-

12 Los intercambios articulan lo que pudiéramos denominar "vertebración territorial", esto es, el conjunto de intercambios territoriales que indican los lazos y relaciones que cohesionan funcionalmente un sistema, lo que a su vez estaría imprimiendo cierta aproximación diferencial de un espacio con respecto a otros (Juárez, 2001). 13 En este contexto, paralelamente en 2003 se publicó el proyecto CiudadValleCentral, de la Escuela de Arquitectura de la Universidad de Talca (CVC, 2003), que sugería examinar las nuevas posibilidades que pudiera generar una macroregión. Esto a partir de un hecho aparentemente menor, su tamaño. Pues un mercado de 900.000 personas, que es más o menos la población de cada región por separado, podría llegar a un mercado real de 3.000.000 de personas, que es la suma de las tres regiones. Este simple hecho implica un cambio de paradigma que entrega posibilidades insospechadas. Finalmente, se establecía, en la misma lógica, el aborde de estrategias comunes en distintos ámbitos, como el portuario, aeroportuario, turístico, productivo y las relaciones con regiones adyacentes de países vecinos. 
cos compartidos y, finalmente, una productividad similar, la cual es intensiva y especializada en recursos naturales. En definitiva, estamos ante un área "suprarregional" (VI, VII y parte de la VIII Región, provincia de Nuble), donde los principales centros poblados que vertebra este territorio tienen entre 30.000 a 200.000 habitantes por ciudad y conforman un corredor central, conectados a la más importante infraestructura chilena, la ruta $5 \mathrm{CH}$ (panamericana sur).

Una característica preliminar es que VCC no tiene ni puerto, ni aeropuerto. Entonces depende fuertemente de sus conexiones extraterritoriales para la trazabilidad de su producción.

Volviendo a la estructura de sus asentamientos y refiriéndonos a esta "caravana", se encuentran ciudades intermedias que manifiestan dinámicas de crecimiento incluso mayores a las de la metrópolis Santiago ${ }^{14}$, siendo enclaves-funcionales administrativos, de servicios y tanto de la elaboración como de distribución nacional e internacional de productos primarios.

Si tomamos en cuenta el número total de habitantes de VCC, la suma sería algo así como 2.100.000, repartidos en 80 comunas, lo que paradójicamente es más que la cantidad de habitantes que tiene una aglomeración de segundo orden en Chile (Gran Valparaíso, Gran Concepción). Pero evidentemente la distribución de estos habitantes es lo que cambia el escenario. Pues en el concierto de las 80 comunas obtenemos que 1.406.972 habitantes se emplazan a lo largo del eje central, es decir, un 66,04\% de la población total de VCC, mientras el resto se dispersa en extenso por el territorio.

Siendo 34 las comunas del eje central, un $42,5 \%$ del total de comunas aglutina el $66 \%$ de los habitantes (Figura 2.1). Este simple hecho parece prefigurar fuertemente las características del patrón de distribución de población, pues sus dinámicas tienden a la concentración sobre el eje central, dejando hacia los bordes del territorio asentamientos menores y menos dinámicos. El escenario anterior se logra completar cuando queda en evidencia la correlación que existe entre las poblaciones urbanas y rurales de las comunas en cuestión, pues las comunas del ámbito central muestran en promedio un porcentaje de ruralidad entre un $10 \%$ o un $20 \%$, mientras que las comunas más alejadas y de mayor superficie lo evidencian entre $50 \%$ y $90 \%$.

Debido a lo anterior, y según los patrones de la distribución de la población localizada en VCC y los niveles de concentración (Figura 2.2), podemos constatar tres niveles distintos de asentamientos con dinámicas particulares. Existen ciudades o nodos principales, que son generalmente capitales provinciales o regionales. Ellas funcionan como unidades espacio-funcionales que tienen la posibilidad de emprender dinámicas propias y sostenidas, reflejadas en niveles de concentración y crecimiento, y que además logran una alta jerarquía en cuanto a los niveles de dotación de bienes y servicios. En un segundo nivel existen pueblos o ciudades menores, que por su cercanía con la entidad principal parecen establecer fuertes interrelaciones; éstas, a su vez, demuestran altas tasas de crecimiento, como veremos más adelante.

Por último, existe una gran cantidad de pequeños poblados, aldeas y caseríos dispersos en gran parte del territorio a mayores distancias del área central. Estas entidades están cerca de grandes áreas cultivables, áreas naturales, o simplemente áreas abandonadas, que pudiéramos caracterizar como los bordes de la suprarregión. Lo que paralelamente demuestra un alto porcentaje rural en el territorio y consecuentemente mucha superficie de áreas desprotegidas, por ejemplo, en términos de planificación.

14 La tasa de crecimiento de Santiago es de 14\%, mientras el país presenta una tasa de $13 \%$. Rancagua 15\%, Curicó $19 \%$, Talca 20\%, Chillán 1\%, por nombrar algunas de estas ciudades (INE, 2002). 
FIgURA 2 | Valle Central de Chile, comunas del eje central y diagrama de centros poblados según habitantes

Rancagua 206.971
Rengo 30.891
Santa Cruz 20.691
San Fernando 49.519
Curicó 93.447
Molina 27.203
Talca 191.154
Constitución 33.914
San Javier 20.524
Cauquenes 30.771
Linares 65.133
Parral 26.397
San Carlos 29.359
Chillán 165.528

FUENTE ELABORACIÓN PROPIA A PARTIR DE CARTAS IGM, ESC: I:IO0.000, INE 2002.

\section{Región del Maule}

Para determinar y verificar lo anterior, creemos necesario, primero, caracterizar las áreas más dinámicas o centrales. Segundo, las áreas o pueblos cercanos y las interrelaciones que generan y, tercero, verificar las áreas más alejadas de estos centros, tomando en cuenta sus funcionamientos y dinámicas. Por lo tanto, nos permitimos acotar el área de estudio, reduciéndola a la región del Maule. Lo anterior nos sirve en forma doble, por un lado, en atención a que la información disponible casi en su totalidad está estructurada en función de las regiones y, por otro, porque esta región pertenece al área central del territorio VCC (Figura 3).

\section{FIGURA 3 | Región del Maule, desplazamientos y población - localización}

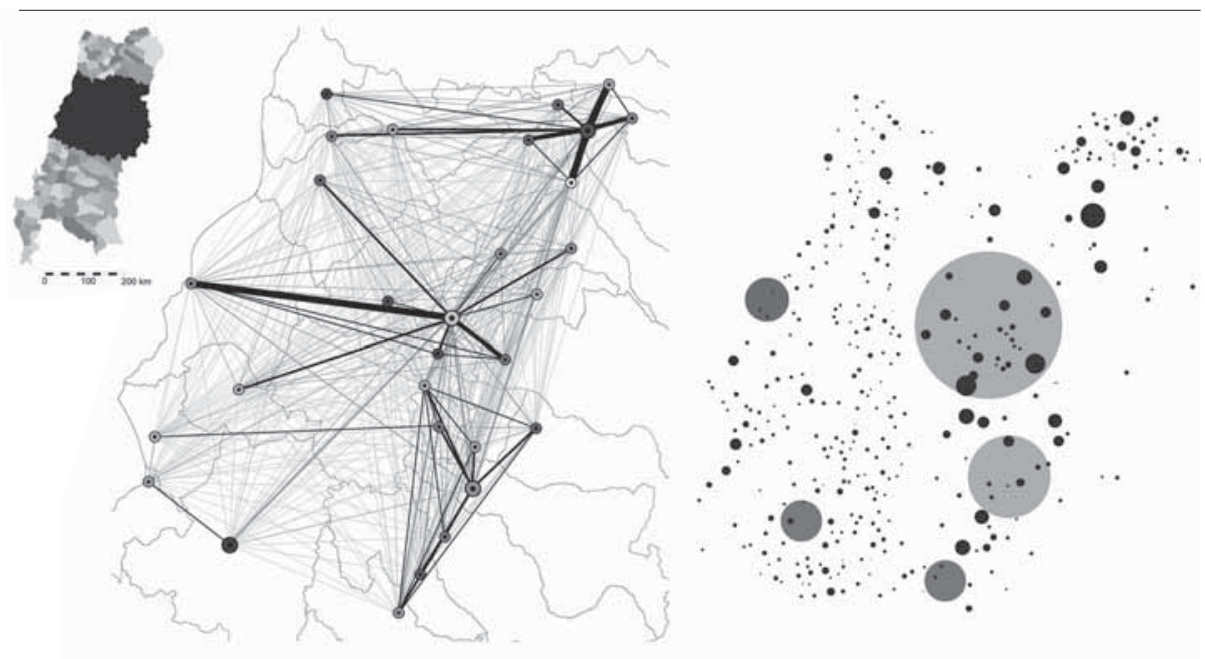

FUENTE ELABORACIÓN PROPIA A PARTIR DE INE, 2000. 
Como es de esperar, la población urbana de esta región se concentra preferentemente a lo largo de la Ruta Central 5 Sur, en unas pocas ciudades principales, capitales provinciales o regional, que suman 525.530 habitantes y representan el $57,9 \%$ de la población urbana total, coherentemente con lo que sucede en VCC. El resto de la población se dispersa a lo largo y ancho del territorio regional en innumerables aldeas, villorrios y centros menores que casi superan los 5 mil (Cuadro 1).

CUADRO 1 | Categoría y población

\begin{tabular}{l|c|c|c}
\hline Categoría & $\mathrm{N}^{2}$ entidades & $\mathrm{N}^{2}$ habitantes & \% población \\
\hline Ciudad & 15 & 525.530 & 57,9 \\
Pueblo & 35 & 77.490 & 8,5 \\
Aldea & 184 & 52.246 & 5,8 \\
Caserío & 853 & 29.429 & 3,2 \\
Otras & 3.993 & 223.402 & 24,6 \\
TOTAL & 5.080 & 908.097 & 100,0 \\
\hline
\end{tabular}

FUENTE INE, 2000.

Las áreas más dinámicas o sistemas de ciudades de esta región se pueden verificar a partir de dos informaciones complementarias, las interrelaciones y los crecimientos. Las primeras, a partir de los viajes diarios o semanales que se realizan, esto tomando como base el último censo de población (INE, 2002) ${ }^{15}$; seguidamente verificaremos los crecimientos poblacionales.

Así, los sistemas centrales -y nos referimos a la ciudad puesta en el eje central, con respecto a las ciudades o pueblos cercanos- a una distancia promedio entre 30 a 45 kilómetros generan un área de relaciones e intercambios bastante intensa. De hecho, a partir de un $15 \%$ y hasta un $20 \%$ de la población económicamente activa de cada ciudad o pueblo dispuesto en esta área, trabaja o estudia en la ciudad principal, con una movilidad diaria o semanal (Figura 3). Esto debido a que estas ciudades agrupan las dotaciones de mayor jerarquía de bienes y servicios, por ejemplo, judicial, salud, educación. Asimismo, estos pueblos o ciudades, que generalmente son cabezas de comunas, muestran, por un lado, crecimientos incluso superiores a los de la ciudad principal, es decir, han podido integrarse a las dinámicas centrales. De esta manera cohesionan funcionalmente el sistema, organizando un área funcional, cuyas dinámicas no necesariamente son congruentes a su división administrativa.

Así, por ejemplo, tomando las dos ciudades más importantes del sistema, Talca y Curicó (Cuadro 2), y sus sistemas de ciudades (no las comunas) se demuestran las altas tasas de crecimiento en casi todo el sistema, e incluso algunos puntos con crecimientos mayores a la ciudad central. Muchos de ellos son estimulados por las dinámicas de aglomeración, enlazándose a las cadenas productivas, al acceso a los proveedores, a los servicios, a la infraestructura o al acceso a centros de estudios superiores, centros de innovación, y distintas ofertas de residencia y, en fin, en busca de activos que hacen que las aglomeraciones tengan unos tamaños mayores a la ciudad central.

Es así como el tamaño de lo que podríamos llamar el Sistema Curicó, es decir Curicó como ciudad central y seis pueblos capitales de comuna, es de 183.993 habitantes. De la misma forma, el tamaño del sistema Talca corresponde a 344.366 habitantes, esta vez tomando la población de las comunas completas.

15 Durante el último censo de población 2002 del Instituto Nacional de Estadística (INE, 2002), se preguntó: “¿Dónde estudia o trabaja?” A partir de esta información y usando plataforma SIG, Arc View 10, se establecieron líneas (grosores) que pudieron finalmente conformar un mapa de estos desplazamientos. Se complementaron con datos del mismo censo para lograr localizar la información. El resultado se muestra en la Figura 3. 
CUADRO 2 | Crecimiento ciudades del sistema

\begin{tabular}{c|c|c|c|c}
\hline \multirow{2}{*}{ Sistema ciudad } & Ciudades & $\begin{array}{c}\mathbf{N}^{\mathrm{a}} \text { hab. Ciudad } \\
2002\end{array}$ & $\begin{array}{c}\mathbf{N}^{\mathrm{h}} \text { hab. Rur.2002. } \\
\text { Comuna }\end{array}$ & $\begin{array}{c}\text { Tasa crecimiento } \\
92 \text {-02 Ciudad }\end{array}$ \\
\hline \multirow{5}{*}{ SISTEMA CURICÓ } & Curicó & 93.447 & 19.079 & $20 \%$ \\
& Rauco & 3.114 & 5.472 & $22 \%$ \\
& Romeral & 3.675 & 9.032 & $48 \%$ \\
& Sagrada Familia & 2.910 & 14.609 & $27 \%$ \\
& Molina & 27.203 & 10.289 & $57 \%$ \\
& Teno & 6.729 & 18.867 & $25 \%$ \\
\hline \multirow{5}{*}{ SISTEMA TALCA } & Talca & 189.505 & 9.995 & $10 \%$ \\
& San Clemente & 13.398 & 28.132 & $24 \%$ \\
& Pencahue & 2.037 & 6.319 & $79 \%$ \\
& Pelarco & 1.822 & 14.148 & $16 \%$ \\
& San Javier & 20.524 & 18.781 & $25 \%$ \\
& Maule & 3.967 & 9.501 & $52 \%$ \\
& San Rafael & 3.482 & 4.117 & $17 \%$ \\
& Cumpeo & 2.651 & 10.074 & $5 \%$ \\
\hline
\end{tabular}

FUENTE ELABORACIÓN PROPIA A PARTIR DE INE I992-2000.

Por otro lado, las ciudades capitales del sistema, como ya apuntamos, muestran un gran dinamismo, creciendo a tasas mayores incluso que la capital nacional (Santiago) e incrementado su consumo de suelo entre un $50 \%$ y un $60 \%$ en los últimos 20 años, ampliando su superficie en las mismas cifras ( $50 \%$ y 60\%) en las proyecciones que se han hechos en los respectivos planes reguladores. Finalmente, se verifica un patrón de crecimiento discontinuo en extensión en el sistema total, pues se muestra un crecimiento orientado hacia la ciudad principal o hacia las infraestructuras mejor desarrolladas y hacia los centros de interés productivos, es decir, a las áreas intermedias entre los centros poblados, lo que finalmente decanta en un área de crecimiento muchas veces sin organización previa (Figura 4).

La aglomeración, vista ahora a partir de sus proyecciones de crecimiento, entrega cifras bastante importantes, casi comparables con las proyecciones de crecimiento de la región completa, que llega a 1.000.000 de habitantes para 2020 (INE, 2002). Lo que nos lleva a afirmar que, según las proyecciones del actual modelo y sin mediar cambios sustanciales los crecimientos futuros, el crecimiento se dará casi en su totalidad en las ciudades más atractivas, por tanto, en el área central (Cuadro 3).

Referido a lo anterior, la literatura ceñida al estudio de sistemas de ciudades nos entrega un dato bastante significativo en torno a lo que llaman "modelos de ciudad central" 16 , donde se examinan sistemas jerárquicos funcionales. El modelo ordena los centros urbanos de una región para establecer jerarquías de tamaño que, a su vez, reflejan la existencia de una jerarquía de bienes y servicios, lo que muestra "que el tamaño del mercado de cualquier bien y servicio tiene un límite máximo como mínimo" (Boix, 2000, p. 226). Es decir, se establecen radios máximos para cualquier bien o servicios, lo cual deja dos conclusiones pertinentes: por un lado, la necesaria aparición de centros alternativos que puedan cubrir el territorio y, finalmente, los tamaños críticos de estos bienes y servicios.

16 Los modelos de ciudad central tienen su origen en el modelo de Christaller (1933), que examina un sistema jerárquico funcional de lugares centrales, sobre una llanura homogénea (mercados sobre diagrama hexagonal). Lo fundamental es el establecimiento de una jerarquía de tamaños, que refleja, a su vez, una jerarquía en los bienes y servicios de una región; así, el tamaño de cualquier mercado de un bien o un servicio tiene un límite máximo y otro mínimo. Por su parte, August Lösch en los años cuarenta nos presenta una versión más formal y explícitamente basada en consideraciones más económicas. 
FIGURA 4 | Región del Maule, Talca crecimiento urbano - proyecciones

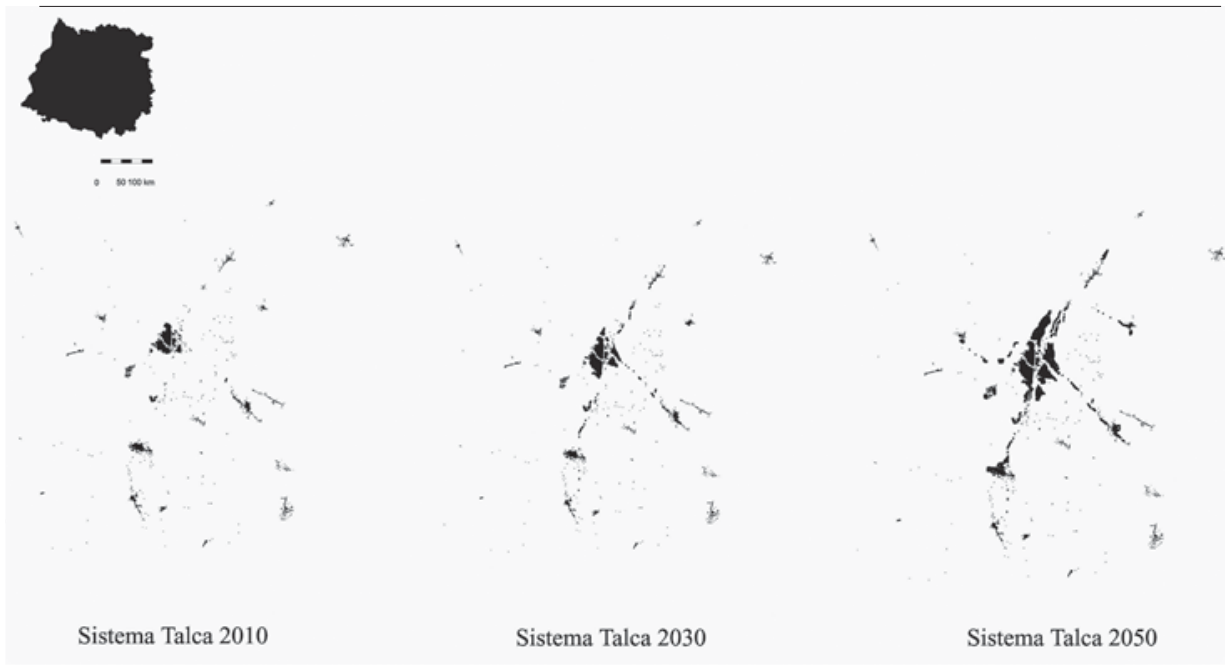

FUENTE ELABORACIÓN PROPIA A PARTIR DE INE 2000/DIAGNÓSTICO PLAN REGULADOR DE TALCA 2008/NO PUBLICADO.

CUADRO 3 | Concentración urbana y proyecciones de población

\begin{tabular}{l|c|cc}
\hline $\begin{array}{c}\text { Centro poblado } \\
\text { provincia }\end{array}$ & $\begin{array}{c}\text { \% concentración/ } \\
\text { Pob. urb provincia }\end{array}$ & 2002 & $2030-2050$ \\
\hline Curicó & $66 \%$ & 77.733 & $236.000-711.333$ \\
Talca & $70 \%$ & 159.711 & $474.711-1.419 .711$ \\
Linares & $63 \%$ & 59.658 & $173.058-513.258$ \\
Cauquenes & $35 \%$ & 27.405 & $72.905-209.405$ \\
\hline
\end{tabular}

FUENTE ELABORACIÓN PROPIA A PARTIR DE INE 2002.

El tercer proceso a caracterizar está representado por pueblos o villorrios que están fuera de la aglomeración, y su patrón de asentamiento está representado por áreas dispersas, que constituyen el $24,6 \%$ de la población regional. Se ubican, generalmente, cerca de áreas de interés natural, antiguas áreas de explotación -como es el secano costero-, al pie de monte o vecinas a áreas de alta productividad. No cabe duda que es tremendamente significativo que el Cuadro 1 de "Categoría y población” (INE, 2002) se refiera a estas áreas como "otros". Esto puede deberse al desconocimiento del tipo de hábitat o a la difícil caracterización de su modelo.

Dentro de los llamados "otros", como dijimos, existen asentamientos de patrón disperso, pequeños y antiguos pueblos, villorrios y asentamientos individuales, todos ellos emplazados sobre una fragmentada matriz de propiedad, estructurada a partir de una red de caminos y senderos. Son áreas fuertemente conectadas a los recursos naturales, donde aparecen nuevos actores: talleres, pequeñas fábricas, turismo, servicios, etc., los que en conjunto provocan que el área ya no dependa sólo de la agricultura ${ }^{17}$. Pero, en lo fundamental, nos enfrentamos a "los nuevos escenarios de la ruralidad o nueva ruralidad" (Canales, 2005, p. 35).

Es en estas grandes dimensiones territoriales donde concurren desequilibrios bastante profundos entre los nuevos y viejos actores territoriales. Es decir, entre los habi-

17 El nuevo sentido de la oportunidad comienza a redirigir de modo significativo la infraestructura existente. Así ocurre, por ejemplo, con el sistema de regadío, que ha hecho posible la inusual presencia de algunas empresas salmoneras, ansiosas de aprovechar el caudal y la temperatura de las aguas para iniciar el proceso de su emblemático producto. 
tantes rurales, pequeños productores y las nuevas productividades exitosas de las regiones especializadas. Entonces, en vastas áreas del territorio, asociado generalmente a fragmentos más pequeños de la matriz, se percibe escasa productividad y una economía no articulada sectorialmente, además de muy bajos niveles de capitalización, baja o nula innovación y baja productividad; por tanto, empobrecimiento y despoblamiento por intensos procesos migratorios. Es así como los ámbitos rurales conforman los últimos niveles de las estructuras territoriales (Arenas, 1999).

Sin embargo, constatamos un tejido reticular donde se logran ciertas jerarquías y puntos de convergencia social.

Es decir, un tejido rural compuesto también de distintas jerarquías, donde los pueblos, generalmente estimulados por la infraestructura, aparecen como integradores a partir de los servicios básicos; luego, sobre el tejido de senderos y caminos, aparecen concentraciones aun menores, donde se van desdibujando las interrelaciones, para concluir en la "unidad productiva". Todo este sistema o tejido son el escenario donde se mueven los viejos y nuevos actores, que demuestran baja interrelación y distancias culturales y económicas importantes, cuyos escenarios nos parecen desconcertantes (Maragaño, 2008).

\section{Los desafíos regionales y la reorganización del territorio}

El gran desafío para las regiones es seguir desarrollándose. En el caso de las regiones altamente especializadas, el desarrollo, aparentemente, está ligado a producir mayor valor agregado a sus productos, incorporar mayor volumen de producción, abrir nuevos nichos de mercado, tanto internos como externos, e incorporar nuevos frentes productivos. Para ello se necesita incrementar la innovación, la infraestructura física y social, la atracción de capital, entre otros factores (De Mattos, 1996).

Según lo analizado, estos factores pueden depender en buena medida de la organización de los procesos de aglomeración productiva y la concentración urbana, los cuales, a partir de ciertas estrategias de conjunto, pueden incrementar su atractivo para desarrollar más innovación que estimule al medio a generar otras oportunidades de desarrollo, así como mejorar la calidad de sus espacios habitados. Por otro lado, la incorporación de las áreas rezagadas puede aportar más agentes a los procesos de desarrollo regional, incrementando su atractivo locacional. Esto último debe llevarse a cabo siempre desde una mirada endógena, complementaria a las miradas exógenas, mejorando la vertebración territorial y, por ende, generando mayor equilibrio en el territorio, a través de la mejora sustancial de la calidad de vida de los habitantes de zonas remotas o "frontera".

Por otra parte, los riesgos de no hacerlo estarían ligados a la generación de áreas ambientales críticas o a una concentración descontrolada, con un colosal consumo de suelo y, finalmente, la generación de deseconomías. De cierta forma, la aglomeración intensiva está en detrimento del crecimiento de otras áreas de la región, y la no vertebración territorial apuntaría a mantener en el subdesarrollo amplias áreas de la región. Como apunta Boisier (2008) citando a Allen J. Scott, "las economías nacionales comienzan a parecerse más y más a confederaciones liberadas de economías regionales" (Scott, 2001). Planteadas así las cosas, la construcción de la región, como objetivo, aparece como un proyecto conjunto y complementario. 


\section{La aglomeración en torno a los recursos naturales, redes, microrregiones y corredo- res alternativos}

Parece una contradicción tratar de impulsar zonas que presentan tales desventajas, en tanto la forma actual del crecimiento se consolida en las "grandes" aglomeraciones fomentadas por la concentración espacial, como fue demostrado anteriormente. Sin embargo, este planteamiento entiende que en virtud de las actuales condiciones de desequilibrio económico y territorial, una vía para alcanzar un desarrollo integral y equitativo es el reconocimiento de áreas potenciales no exploradas y difícilmente reconocidas desde la lógica imperante, asumiendo las ventajas de la modernidad.

Así, hoy nos parece fundamental incorporar a los procesos de estudio y desarrollo la variable territorio, como es el razonamiento de Becattini ${ }^{18}$ (2006), quien señala que para entender las dinámicas de la producción no basta con estudiar las dinámicas de la empresa como sector, sino que se debe abordar el territorio donde las empresas operan, lo que integra nuevos fenómenos además de los paradigmas económicos clásicos. Bajo este enfoque, las variables geográficas -o espaciales- e históricas ya no son sólo un soporte de la actividad, sino las posibles portadoras de externalidades positivas (Trullén, 2002). Esto implica incorporar a la variable territorial como factor productivo, lo que abre nuevas posibilidades.

Es así como hoy es posible establecer ciertas alternativas a las economías de aglomeración o concentración, es decir, una oportunidad para el resto del territorio. Lo anterior, en cuanto entendiéramos que los llamados "rendimientos crecientes", pilares de la comprensión de las economías de aglomeración, pudieran comportarse de formas aún más dinámicas (Fujita, M.; Krugman, P. \& Venables, A.J., 2000). Así, las economías en red se transforman en una herramienta de gran utilidad, en la medida en que impiden que los análisis pongan a la ciudad-estrella en el centro, ocupando este lugar la red de ciudades, compitiendo con éxito con las metrópolis concentradoras (Trullen, 2002). La colaboración entre el sistema de redes de ciudades parece ser el paradigma, mediante la infraestructura, corredores de transporte y las telecomunicaciones, aun cuando estos factores no aseguran la existencia de una red. En este caso, parece necesario incluir una definición más flexible, que pudiera ajustarse a la realidad territorial de regiones especializadas. Dematteis (1990) señala que existe la posibilidad de que coexistan estructuras jerárquicas tipo cristalleriano y otras de otro tipo, lo que posibilita una armadura o vertebración territorial en diferentes niveles no excluyentes, lo que define que las redes territoriales son estructuras espaciales de diversos tipos, abriéndose la posibilidad de pensar en la constitución de redes e intercambios donde existe la posibilidad de ampliar o sumar actores y volúmenes al desarrollo del territorio.

En este caso, el argumento no significa que los procesos de autoorganización regional, en términos del mercado como organizador del territorio y las dinámicas capitalistas, no sean útiles o relevantes, sino que además pueden existir ciertas ventajas estratégicas derivadas de visiones "desde abajo hacia arriba". Es por esto que se hace necesario estudiar y seleccionar áreas del territorio que puedan entrar en el contexto de dinámicas multiescalares, como lo demuestra la aglomeración exitosa, y que puedan incorporar nuevas áreas al desarrollo. Lo que no significa la construcción de una red, sino la incipiente posibilidad de construir relaciones que fortalezcan las interrelaciones.

18 Becattini, con su trabajo "Del sector industrial al distrito industrial" (1979), abre la posibilidad de una nueva teoría económica que sustenta nuevas variables, a partir de su estudio de la ciudad textil del Prato, Italia. Así, plantea que gracias a externalidades dinámicas de naturaleza territorial, era posible generar rendimientos crecientes sin necesidad de integrar verticalmente la producción de grandes empresas (Trullen, 2002). 
Por tanto, la evidencia muestra que es necesario desarrollar nuevas áreas para incrementar la cobertura regional, pues existe una limitación en el tamaño de los mercados, tanto de bienes como de servicios (Boix, 2004), lo cual incide en la área de cobertura de estos agentes. Es decir, a ciertas distancias es necesario generar nuevos nodos que pudieran integrar estos bienes y servicios, y que incluyan a las nuevas poblaciones que los demanden.

También es correcto insistir que entendemos que las aglomeraciones pequeñas que pudieran generar dinámicas en estos territorios, son generalmente "articulaciones marco, tanto públicas como privadas, lideradas por iniciativas estrategias competitivas en los mercados" (Potter, 2004, citado por CEPAL, 2006, p. 136). Esto, según la literatura especializada, entrega lineamientos que se complementan con los anteriores, amparados en la evidencia de que los efectos frontera -que anteriormente se comentaronhan podido ser superados por las distintas posibilidades que entrega la inserción económica. Lo anterior, en tanto que estas zonas frontera pudieran iniciar o desarrollar conexiones multiescalares, conectándose directamente con mercados internacionales a partir de la infraestructura esencial para que estos conglomerados funcionen (CEPAL, 2006). Para ello se requiere desarrollar nuevas herramientas basadas en la redefinición de las relaciones "macro-meso-micro", reconociendo a "las regiones y localidades como partes de sistemas económicos, redes y flujos de recursos más amplios, antes que como unidades autocontenidas y cerradas" (Turok, 2004).

Por tanto, entendemos que las lógicas permiten leer variables más flexibles y dinámicas, donde los factores productivos puedan actuar de forma integrada y abierta, y en las cuales las economías de localización y las ventajas competitivas juegan un importante papel.

En Chile durante los años 70 se definió una metodología y políticas referidas a microregiones (Conara, 1976) ${ }^{19}$ pues se hacía necesario definir ámbitos más pequeños en la organización del proceso de regionalización que en esos años se llevaba a cabo en Chile. Este

FIGURA 5 | Conexiones microrregionales. Diagrama y ejes alternativos

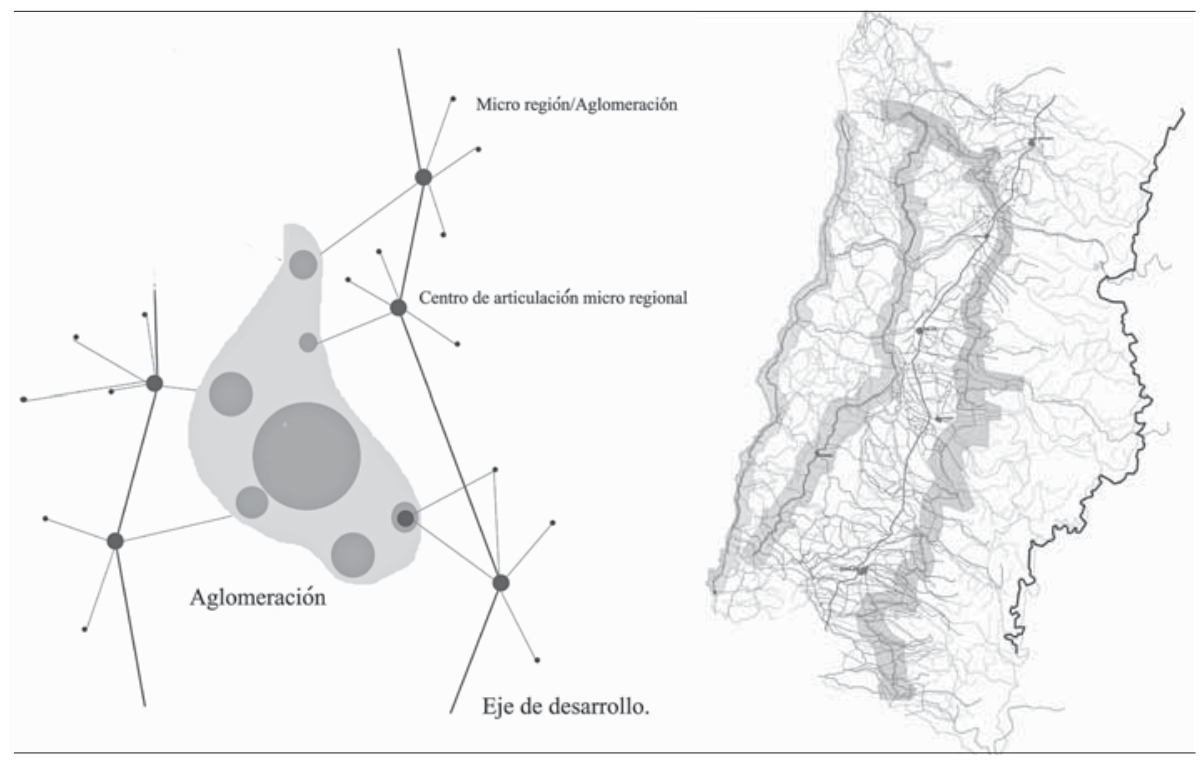

FUENTE ELABORACIÓN PROPIA.

19 Generalmente el concepto microrregión es entendido como un área geográfica de particularidades, sobre todo en trabajos dedicados a lo agroproductivo. Quisiéramos entenderla como una zona geográfico-social, en cuya definición intervienen elementos topográficos, ecológicos y poblacionales, los cuales le imprimen una dinámica propia. 
proceso, nos parece, se focalizaba en las especificidades productivas de cada microrregión, es decir, la metodología partía de potenciar sus condiciones agroclimáticas y geográficas para definir aquella porción de territorio que pudiera tratarse específicamente.

A partir de aquí, nos propusimos definir microrregiones, pero esta vez a partir de sus sistemas de interrelación, es decir, a partir del estudio de la "concentración de funciones" en una localidad que pudiera generar cierta articulación hacia el conjunto y formar parte de un determinado nivel de la jerarquía territorial; siendo relevante, por un lado, conocer la propia dinámica que tienen los lugares centrales, pues depende de su capacidad para proporcionar bienes y servicios tanto a su población como a la población residente en otros asentamientos, principalmente en aquellos que forman parte de la microrregión de la cual se constituyen como "lugar central".

Lo que se buscó fue ubicar puntos que pudieran servir como áreas multiescalares comprometidas con los servicios más importantes atendiendo al sistema y, por otro lado, que estos puntos puedan integrarse a potencialidades mayores, como áreas cultivables o naturales, desde donde se estimule el dinamismo socioeconómico, el acceso a ciertos servicios y equipamientos, el emprendimiento de dinámicas propias, el acceso a diferentes sistemas productivos y el mejoramiento de sus capacidades de interacción con otros asentamientos de igual jerarquía o, finalmente, con los complejos urbanoregionales. Todo lo anterior, con el propósito de que se pueda contribuir a implementar un modelo de desarrollo cuyo objetivo sea disminuir las diferencias en los niveles de las estructuras territoriales mediante un proceso de integración regional a mayor escala.

Ahora, según esta intención, logramos visualizar algunos asentamientos que funcionarían como nodos de integración, a distintas escalas (Cuadro 4). A partir de sus características y capacidades, establecimos criterios de discriminación (multicriterio), lo cual sumado a los criterios de localización clásica (buffers) y posibilidades de integración construyeron áreas o microrregiones.

Lo paradójico al intentar generar este análisis de las capacidades microrregionales y sus integraciones, es que conectando áreas de igual jerarquía, es decir, conectando áreas microrregionales con otras, en teoría, se configura una serie de corredores longitudinales. Resultantes de las condiciones geográficas y posibilidades reales físicas que entrega el territorio, se desarrollan áreas alternativas al corredor central, que finalmente adquieren la escala de toda la macrorregión VVC (Cuadro 4) (Figura 5).

Así, y desde la construcción de microrregiones, se produce la apertura de la Región del Maule y la conexión de todo el VCC, lo que permite pensar en nuevos corredores y ejes de desarrollo, a lo largo de los cuales se desarrollen aquellas localidades dispersas y aisladas, en los bordes del territorio y alejadas del Eje Central. Es así como en cada uno de estos corredores se distribuye una población que sobrepasa los 200 mil habitantes, y a lo largo de los cuales es posible identificar en la actualidad algunos centros que, por suposición estratégica y niveles de equipamiento y servicios, logran concentrar una mayor cantidad de población y ser focos de atracción para las localidades menores, dispersas en su alrededor, y cumplir con las relaciones multiescalares que demandan la conformación de estas aglomeraciones.

La superposición de nuevos ejes y los actuales ejes bioceánicos (ejemplo: Paso Pehuenche, Paso Vergara) y la estructura de filamentos o tejidos rurales, constituye una red de distintos grosores e intensidades, lo que posibilita la integración más rápidamente de áreas hoy día rezagadas.

De todo lo que se ha señalado, una mejor vertebración microrregional también ayudaría a romper con los moldes restringidos y obsoletos que impone la actual estructura político-administrativa, que hace que la comuna se aísle en lugar de crecer e integrarse 
a otras para fortalecerse y establecer relaciones de carácter intercomunal, interregional e internacional. De esta forma, se favorecería a todo el país, al no quedar desconectada del resto en situaciones tales como el colapso de la única vía expedita que dispone en la actualidad para conectarse desde el norte y centro del país al centro-sur y sur del mismo, vía terrestre. Esto equivale a realizar un ejercicio de planeación aplicada desde los niveles inferiores, contrario a la forma tradicional de llevar a cabo los procesos de planeación desde las esferas macrorregionales.

CUADRO 4 | Tabla metodología discriminación áreas de articulación

\begin{tabular}{|c|c|c|}
\hline \multicolumn{3}{|c|}{ IDENTIFICACIÓN (DISCRIMINACIÓN) } \\
\hline Producción (Áreas) & Capacidades & \\
\hline $\begin{array}{l}\text { Vitivinicultura } \\
\text { Hortof ruticultura } \\
\text { Ganadería } \\
\text { Arroz y cereales } \\
\text { Forestal e industria } \\
\text { secundaria de la madera } \\
\text { Turismo } \\
\text { Industria } \\
\text { Áreas de protección y } \\
\text { reservas }\end{array}$ & $\begin{array}{l}\text { Población. } \\
\text { Cobertura ( } \text { buffer) } \\
\text { Servicios y equipamientos } \\
\text { existentes. } \\
\text { Productividad del trabajo } \\
\text { (rendimiento) } \\
\text { Localización (buffers) }\end{array}$ & $\begin{array}{l}\text { Nivel de empleo/desempleo } \\
\text { Organización } \\
\text { Ruralidad e inf raestructura } \\
\text { de apoyo (redes de servicios) } \\
\text { y sistema de centros poblados } \\
\text { (concentración/dispersión) } \\
\text { Mercado (nacional/internacional) }\end{array}$ \\
\hline \multicolumn{3}{|c|}{ ORGANIZACIÓN SEGÚN FRANJAS O EJES LONGITUDINALES } \\
\hline Ubicación, tramos & Organización espacial (tipología) & Centros poblados \\
\hline $\begin{array}{l}\text { Arco Oriente } \\
\text { (Cordillera y Precordillera) }\end{array}$ & Lineal Longitud. & $\begin{array}{l}\text { Romeral-Cumpeo-Colbún-Catillo- } \\
\text { Coihueco-Pinto. }\end{array}$ \\
\hline Eje Central (Ruta 5 Sur) & Radiocéntrica & $\begin{array}{l}\text { Curicó (Romeral, Teno, Rauco, } \\
\text { Sagrada Familia, Lontué, Molina), } \\
\text { Talca (San Rafael, Pelarco, San } \\
\text { Clemente, Maule, Pencahue), } \\
\text { San Javier (Yerbas Buenas, Villa } \\
\text { Alegre), Longaví, Parral }\end{array}$ \\
\hline $\begin{array}{l}\text { Arco Poniente } \\
\text { (Depresión Intermedia, } \\
\text { Cordillera de la Costa) }\end{array}$ & Lineal Longitud & $\begin{array}{l}\text { Marchigüe, Lolol, Hualañé, Colín, } \\
\text { Gualleco-Pencahue, Corinto, Sauzal, } \\
\text { Cauquenes, Quirihue, Treguaco, } \\
\text { Coelemu }\end{array}$ \\
\hline Borde Costero & Lineal Longitud & $\begin{array}{l}\text { Pichilemu, Llico, Vichuquén, } \\
\text { Curepto, Constitución (Putu, Las } \\
\text { Cañas), Chanco-Pelluhue, Curanipe, } \\
\text { Cobquecura, Tomé }\end{array}$ \\
\hline Corredores Bioceánicos & Lineal Transversal & Paso Vergara, Paso Pehuenche \\
\hline
\end{tabular}

CUADRO 5 | Concentración urbana y ejes del desarrollo

\begin{tabular}{|c|c|c|}
\hline Eje & Tramo & $\mathrm{N}^{\mathrm{p}}$ Habitantes \\
\hline 1 Borde Costero & Pichilemu-Tomé & 213.677 \\
\hline $\begin{array}{l}2 \text { Secano Costero } \\
\text { (ruta del vino y la madera) }\end{array}$ & Marchigüe-Trehuaco & 206.170 \\
\hline $\begin{array}{l}3 \text { Arco Oriente Norte } \\
\text { (bioceánico Norte, San Antonio) }\end{array}$ & Colbún-Marchigüe & 225.750 \\
\hline $\begin{array}{l}4 \text { Arco Oriente Sur } \\
\text { (bioceánico Sur,Talcahuano) }\end{array}$ & Colbún-Yungay & 167.406 \\
\hline 5 Eje Central & Rancagua -Chillán & 731.741 \\
\hline Total & & 1.544 .744 \\
\hline
\end{tabular}




\section{Conclusiones preliminares}

Hoy nos parece necesario tener en cuenta las fuerzas que dirigen los mercados para realizar correcciones al sistema. Por un lado, plantear alternativas a las economías regionales depende en gran medida de romper con los códigos que sobreviven, a pesar de las transformaciones y nuevas necesidades de nuestras regiones. El fin será siempre el desarrollo, más allá de las demandas sectoriales, lo cual ayuda a asegurar condiciones y calidad de vida adecuadas a los habitantes. Por ello es necesario realizar acciones para avanzar en los procesos de organización territorial y vertebración territorial.

Grandes problemas enfrentan las localidades más alejadas de los centros dinamizadores. Estas entidades, a nuestro entender, representan un reto para el sistema completo en el presente escenario de regiones de alta especialidad. Así, aparece urgente proporcionar una vertebración del territorio y, finalmente, borrar los efectos frontera.

La posibilidad de abrir el territorio, constituyendo distintas redes de interrelación, aparece como una oportunidad que actúe potenciando la vertebración del territorio, aumentando sus capacidades de construcción de sinergias.

La construcción de una estrategia microrregional podrá ayudar a lograr una visión de conjunto de los procesos de urbanización en la entidad regional y, al mismo tiempo, propiciar un cambio en la óptica en la que tradicionalmente se basan las políticas públicas que abordan la problemática del desarrollo urbano-regional.

Para que este procedimiento pueda garantizar resultados positivos se requiere como condición indispensable poner en marcha programas integrales de dotación de servicios, infraestructura, comunicaciones y transportes, fomento a las actividades productivas, así como acciones de conservación y preservación del medio ambiente, los cuales habrán de funcionar como elementos articuladores entre las microrregiones y el conjunto de la región a partir de un planeamiento multiescalar.

Finalmente, nos preocupan los territorios rurales que tradicionalmente van a la zaga de las influencias de los centros urbanos más próximos, por lo que es fundamental precisar, desde una perspectiva sistémica, cuáles son los mecanismos y estrategias que se pueden poner en operación para acumular ventajas de ubicación propicias para la competitividad e intercambio intrarregional. Es decir, debemos producir ideas que estimulen la generación de aglomeraciones en torno a los espacios naturales, sin que necesariamente dichos espacios se conviertan en ciudades o, más bien, en sistemas urbano-territoriales. IEURE

\section{Referencias bibliográficas}

Arenas, F. \& Sabatini. F. (1994). Comunidades territoriales pobres y explotación de recursos naturales. Ambiente y Desarrollo, CIPMA, 10 (3), 36-42.

Arenas, F. (1999). Organización Territorial y Desarrollo Regional en Chile. Instituto de Economia y Geografia, Madrid (C.S.I.C.) 60, (234), 101-119.

Becattini, G. (2006). Vicisitudes y potencialidades de un concepto: el distrito industrial. Economía industrial. (359). 21-28.

Bengoa, J. (Ed.). (2007). Territorios rurales. Movimientos sociales y desarrollo territorial rural en América Latina. Santiago: Rimisop.

Boisier, S. (1996). Modernidad y Territorio. Cuadernos del ILPES, n 42, Santiago de Chile.

Boisier, S. (2004). Desarrollo territorial y descentralización: El desarrollo en el lugar y en las manos de la gente. EURE, 30, (90), 27-40. 
Boisier, S. (2008). Territorio, Estado y Sociedad en Chile. La dialéctica de la descentralización: entre la geografía y la gobernabilidad. Tesis Doctoral, Facultad de Ciencias Económicas y Empresariales Universidad Alcalá de Henares, España. Recuperada el 20 de noviembre de 2009 de: http.//www.eumed.net/tesis/2008/sbe/

Boix, R. (2002). Policentrismo y redes de ciudades en la región metropolitana de Barcelona. En J. Subirats (ed.), Redes, territorios y gobierno. Barcelona, España: Diputación de Barcelona.

Camagni, R. (2005). Economia Urbana. Barcelona, España: Antoni Bosch. S.A.

Canales, M. (2005). Chile rural, un desafío para el desarrollo humano. Temas de Desarrollo Humano Sustentable. La nueva ruralidad en Chile: apuntes sobre subjetividad y territorios vividos. $\mathrm{N}^{\circ} 12$, Santiago: PNUD.

CEPAL (Comisión Económica para América Latina y el Caribe) (1998). Ciudades Intermedias en América Latina y el Caribe: propuesta para la gestión urbana. Ricardo Jordán y Daniela Simioni. (Ed.)

CEPAL (2006). Aglomeraciones en torno a los recursos naturales en América Latina y el Caribe: Politicas de articulación y articulación de politicas (88) Santiago de Chile.

Comisión Europea (1995). Europa 2000+. Cooperación para la ordenación del territorio europeo. Luxemburgo.

Daher, A. (2003). Regiones-commodities: Crisis y contagio en Chile. EURE, 29 (86), 89-108.

De Mattos, C. (2002). Santiago de Chile de cara a la globalización: ¿otra ciudad? Revista de Sociología y Politica, (19): 31-54.

De Mattos, C. (1996). Avances de la globalización y nueva dinámica metropolitana: Santiago de Chile, 1975-1995. EURE, 22 (65), 39-63.

Fernández, V. (2007). Explorando las limitaciones del nuevo regionalismo en las políticas de la Unión Europea: Una perspectiva latinoamericana. EURE, 33 (98), 97-118.

Gault, M. (1989). Villes intermédiaires pour l'Europe? France: Syros Alternatives.

Geisse, G. (1983). Economía y Politica de la Concentración Urbana en Chile. México: El Colegio de México/Pispal.

Fujita, M.; Krugman, P. \& Venables A.J. (2000). Economía espacial. Barcelona: Editorial Ariel.

Juárez Sánchez-Rubio, C. (1996). Europa 2000 +. Cooperación para la ordenación del territorio europeo. Comisión Europea. Luxemburgo. 10(15), 148-150.

Krugman, P. (1997). La organización espontánea de la economia: the Self-organizing economy. Barcelona: Antoni Bosch S.A.

Maragaño, A. (2008). Mariposas, el territorio y la ciudadanía rural. Revista de la Escuela de Arquitectura de la Universidad de Talca, 2, 46-56.

Mertins, G. (2000). Ciudades medianas en América Latina: criterios, indicadores y el intento de un modelo de su diferenciación socio-espacial y funcional. Espacio y Desarrollo, 12: 123-140.

Pulido, N. (2003). Globalización y surgimiento de ciudades “intermedias" en América Latina y en Venezuela. Revista Geográfica Venezolana, 45(1) 91-121.

Roman, J. (2003) (Ed.) CiudadValleCentral. Talca, Chile: Escuela de Arquitectura Universidad de Talca.

Romero, H. \& Toledo, X. (2000). Ecología urbana y sustentabilidad ambiental de las ciudades intermedias chilenas. Anales de la Sociedad Chilena de Ciencias Geográficas: 445-452.

Rovira, A. (2000). Puerto Montt: El paso de una ciudad menor a centro de desarrollo interregional en una ciudad del sur de Chile. Espacio y Desarrollo, 12 83-102.

Sabatini, F. \& Wormald, G. (2004). Santiago de Chile bajo la Nueva Economía, 1980-2000: crecimiento, modernización, y oportunidades de integración social. The Center for Migration and Development, Working Paper Series, Princeton University, CMD Working Paper, n. 04-04a.2.

Scott, A.J. (2001). Les régions et l'economie mondiale. Paris: L'Harmattan.

Szary, A. (1997). Regiones ganadoras y regiones perdedoras en el retorno de la democracia en Chile: poderes locales y desequilibrios territoriales. EURE, 23 (70), 59-78.

Turok, I. (2004). Cities, Regions and Competitiveness. Regional Studies, 38 (9), 1069-1083. 